\title{
Licence Plate Character Recognition Using Artificial Immune Technique
}

\author{
Rentian Huang, Hissam Tawfik, and Atulya Nagar \\ Intelligent and Distributed Systems Lab, Deanery of Business and Computer Sciences, \\ Liverpool Hope University, Liverpool, United Kingdom L16 9JD \\ $\{10076507$, TAWF IKH , NAGARA $\}$ @Hope.ac.uk
}

\begin{abstract}
This paper proposes the application of Artificial Immune Technique in Licence Plate Character Recognition (LPCR). The use of Clonal Selection Algorithm (CSA) is composed of two main stages: (1) dynamic training samples; and (2) a choice of the best antibodies based on the three main clonal operations of cloning, clonal mutation and clonal selection. Once memory cells are established it will output the classification results using Fuzzy K-Nearest Neighbor (KNN) approach. The performance of CSA is compared to the Back Propagation Neural Networks (BPNN) in solving a LPCR problem. The experimental results show that the Artificial Immune Technique has a favorable performance in terms of being more accurate and robust.
\end{abstract}

Keywords: Artificial Immune System (AIS), Clonal Selection Algorithm (CSA), Licence Plate Recognition (LPR).

\section{Introduction}

Licence Plate Recognition (LPR) System combines image processing and character recognition technology to identify vehicles by way of automatically reading their number plates. A typical LPR process consists of three stages: 1) licence plates location, 2) character segmentation and 3) character recognition. LPR demonstrates particularly useful and practical vehicle identification technology as it assumes no additional means of vehicle identity apart from the existing and legally required number plate. Furthermore, when data gathered by an LPR system is stored and organized within a database, more complex information-driven tasks may potentially be performed such as, vehicle travel time calculations as well as border controls. However, in practice it is a very difficult task due to the variety of environmental conditions. LPR system is usually conducted under certain restrictive conditions such as, indoor scenes, stationary backgrounds, fixed illumination, prescribed driveways, limited vehicle speed, and at a designated range of distance between camera and vehicle [1]. Despite these current limitations, LPR finds applications in private parking management, traffic monitoring, automatic toll payment, surveillance and security enforcement [2].

Numerous algorithms have previously been exploited such as, Hidden Markov Models (HMM) [3], Artificial Neural Networks (ANN) [4], Hausdorff Distance [5], 
Support Vector Machine [6] (SVM)-based character recognizer and template matching that leave a lot of room for improvements [7]. The focus of this paper is to investigate a character recognition technique using the Artificial Immune System (AIS) based CSA. A number of adjustments are made to the basic implementation of CSA in order to improve the performance, especially in using a new dynamic training to establish the immune memory (collection of antibodies) for classification. Additionally, Neural-Network results are presented to compare the performance. The experimental results show that CSA for character recognition has a better performance in terms of successful classification of the characters of licence plates. CSA proved more accurate and robust compared to Neural Networks.

The rest of this paper is presented as follows: The LPR architecture in section 2 which includes a review of relevant techniques used for tackling character recognition of LPR. Section 3 introduces our CSA and the features added to it for character recognition. Section 4 provides the experimental details and highlights the compared performance of the CSA in character recognition. Section 5 gives the conclusion and proposes the future work.

\section{Car Plate Recognition}

Car plate recognition algorithms reported in the research are generally composed of three main steps, 1) locating licence plates, 2) segmenting licence numbers and 3) identifying the characters. Fig. 1 illustrates our proposed LPR.

In locating the licence plate, a colour edge detector is developed to detect the type of edges contained within the licence plate. While, multiple licence plate candidates are normally detected, size and shape filtering is used to remove objects that do not satisfy some specific conditions. The target will select regions that serve as possible licence plate boundaries. In order to achieve possible licence plate boundaries the area-to-perimeter ratio of the candidate area is compared with the actual standard ratio of a number plate. Once a licence plate candidate has been extracted from the image, the licence number segmentation preprocessing component will continue to perform three tasks, Grey-level transform, Median filtering and Binarisation. A vertical projection is performed to segment the characters with each character image normalized to a size of $16 \times 16$ after segmentation. Following character segmentation from the plate region a method needs to be selected for character recognition which is the main subject of this work.

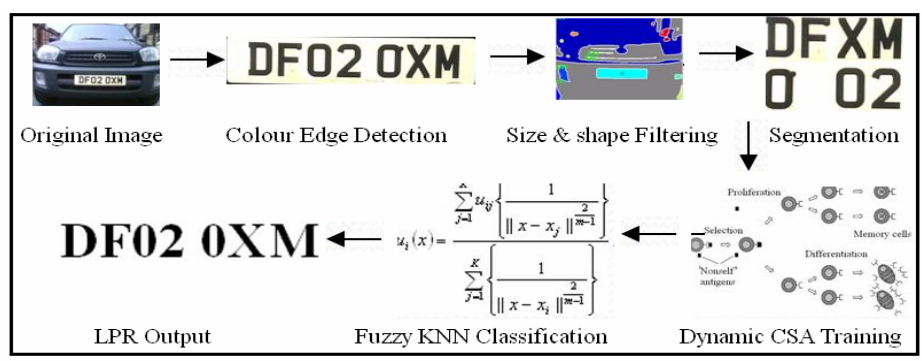

Fig. 1. Diagram of our LPR process 
There has been a large number of character recognition techniques reported. HMM for recognition begins with the pre-processing and parameterization from the region of interests detected in the previous phase. Researchers report that the width of the plate in the image after rescaling lies between $25 \%$ and $75 \%$ for an image width arranging from 200 and 600 pixels. This reveals the necessity for good character analysis when implementing HMM, which places a restriction on the effective distance of the recognition system [3].

Various types of ANN had been used for licence plate character identification such as the work done by Broumandnia and Fathy [8]. A self-organized Neural Network based on Kohonen's Self-Organized Feature Maps (SOFMs) was implemented to tolerate noisy, deformed, broken, or incomplete characters acquired from licence plates, which were bent or tilted with respect to the camera [9]. Probabilistic Neural Networks (PNNs) for LPR were also introduced by Anagnostopoulos et al. [10]. Hausdorff distance is a method for LPCR that compares two binary images. Its main problem is the computational burden. Its recognition rate is very similar to that obtained with Neural-Network classifiers, but it is slower [5]. Kim et al. designed a system implementing four SVMs and report an impressive average character recognition rate. The architecture, however, is strictly designed for Korean plates [6]. A suitable technique for the recognition of single font and fixed size characters is the pattern matching technique. The recognition process was based on the computation of the normalized cross correlation values for all the shifts of each character template over the sub-image containing the licence plate [7].

LPR problem continues to be a challenge for artificial intelligence solutions and novel approaches are therefore needed to improve the performance and efficiency of LPCR algorithm. In this work, an AIS character recognition technique based Clonal Selection Algorithm is presented for solving LPR problem.

\section{Immune Techniques for Character Recognition}

Artificial Immune System is a rapidly emerging technique for developing mechanisms for learning, prediction, memory and adaptation. AIS mimics the biological immune systems as these offer powerful and robust information processing capabilities for solving complex problems [11]. The immune system is a biological pattern recognition and classification system which learns to distinguish the self from nonself. The immune systems behaviour is an emergent property of the entire population of diverse agents and improves performance by weeding out the weakest players, replacing them with agents as different as possible. The immune system is computationally one of the least understood biological paradigms but has drawn significant attention. AIS started to be used in many application domains including computer security, optimization, robotics, data mining, fault detection, anomaly detection, and pattern recognition [12].

\subsection{Clonal Selection}

Clonal Selection Theory, the famous theory in immunology, was put forward by Burnet in 1978 [13]. Its main ideas lie in the fact that the antigen can selectively react 
to the antibodies, which are natively produced and spread on the cell surface in the form of peptides. When cells are exposed to an antigen, the antigen stimulates an immune cell with appropriate receptors to proliferate (divide) and mature into terminal plasma cells. The process of cell division generates a clone, i.e., a set of cells that are the progenies of the single cell. In addition to proliferating and maturing into plasma cells, the immune cells can differentiate into long-lived memory cells. Memory cells circulate through the blood, lymph and tissues, and when exposed to a second antigenic stimulus they commence to differentiate into large immune cells (lymphocyte) capable of producing high affinity antibody preselected for the specific antigen that once stimulated the primary response. Fig. 2 depicts the clonal selection principle.

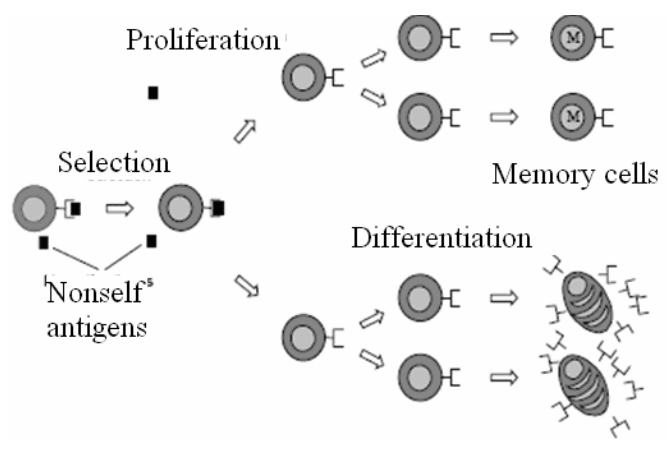

Fig. 2. Clonal Selection Principle

Clonal selection is a dynamic process of the immune system stimulated by the selfadapting antigen. Some biologic features such as learning, memory and antibody diversity can be used in artificial immune systems to solve complex problems. De Castro and Von Zuben proposed the first Clonal Selection Algorithm, called CLONALG and suggested that it could be used for pattern recognition. They generated random antibodies to be used as the target patterns in CSA. They used a set of 12 x 10 binary images as the target patterns [14]. Utpal Garain et al. proposed a CSA used for a 2-class problem to classify pairs of similar character patterns and claimed promising results. Setting aside the classification power, data reduction had been another capability of CSA [15]. Utpal Garain et al. further explored the potential of CSA in pattern recognition by applying the CSA for a 10-class classification problem. Empirical study with two datasets shows that the CSA has very good generalization ability with experimental results reporting the average recognition accuracy of about $96 \%$ [16].

\subsection{Clonal Selection for LPCR}

The proposed clonal selection algorithm for character recognition is composed of two main processes: firstly, selecting samples and training these samples using CSA; secondly, using fuzzy (KNN) approach to output the classification results for LPR. The essentials of clonal selection are established as follows. Antigens are images 
stored in a matrix and represent the licence plate character of the system. Antibodies are the candidates that go through clonal process and try to catch and represent the common features of antigens.

The affinity between antibody and antigen is the reflection of the total combination power located between them. For classification problem, hamming distance (HD) and similarity functions are used to measure affinity between antigen and antibody. The Hamming distance rule is presented below:

$$
\text { difference }=\sum_{i=1}^{n} \sum_{i=1}^{\text {len }} \operatorname{pos}_{i}=A b_{i} \oplus A g_{i}^{j} \quad \text { Affinity }=- \text { difference }
$$

Where $A b_{i}$ is the $i t h$ bit in the antibody $A b, A g_{i}^{j}$ is the $i t h$ bit in the antigen pattern examples $A g^{j}$ and $\mathrm{n}$ is the number of examples for a particular pattern class. Len is the total length of an antibody and $\oplus$ represents the exclusive XOR operator.

Another formula used to measure similarity (affinity) of antigen to antibody interaction is presented as shown in Eqn (2) below:

$$
S\left(A g_{1}, A g_{2}\right)=\frac{1}{2}-\frac{S_{10} S_{01}-S_{00} S_{11}}{2 \sqrt{\left(S_{11}+S_{10}\right)\left(S_{01}+S_{00}\right)\left(S_{11}+S_{01}\right)\left(S_{10}+S_{00}\right)}}
$$

where $\left(A g_{1}, A g_{2}\right)$ are the two matrices to be compared, $S_{10}, S_{01}, S_{00}, S_{11}$, which denote the number of zero matches, one matches, zero mismatches, and one mismatches. The value of $S$ is in the range $[0,1]$, where 1 indicates the highest and 0 indicates lowest similarity between the samples.

In immunology, cloning selects a number of antibodies with the highest affinity and cloning them based on their antigenic affinities. The higher the antigenic affinity, the higher the number of clones will be generated. The total number of clones generated $N_{c}$ is defined in Equation 3 as follow:

$$
N_{c}=\sum_{i=1}^{n} \operatorname{round}\left(\frac{\beta \cdot N}{i}\right)
$$

where $\beta$ is a multiplying factor, $\mathrm{N}$ is the total number of antibodies, round (.) is the operator that rounds its argument toward the closet integer.

In clonal mutation, the clone set $C^{i}$ is used to produce mutated offspring $C^{i^{*}}$. The higher the affinity an antigen has, the smaller the mutation rate. The algorithm for mutation is described in Fig. 3. The Equation is defined as follows:

$$
\Delta(t, y)=y\left(1-r^{\left(1-\frac{t}{T}\right)^{\lambda}}\right)
$$

where $t$ is the iteration number, $T$ is the maximum iteration number, $r$ is a random value in the range $[0,1]$, and $\lambda$ is used to decide the nonconforming degree. 
Find the maximum and minimum in population $C^{i}$.

For each $A b$, do

Generate a random value in the range $[0,1]$, named $\mathrm{mr}$

Generate another random value in the range $[0,1]$, named t0

If $\mathrm{mr}<$ mutation_rate

If $\mathrm{t} 0>=0$

$A b=A b+\Delta(t, \max -A b)$

else $A b=A b-\Delta(t, A b-\min )$

return $A b$

Fig. 3. Mutation rate control algorithm

The final operation is clonal selection which includes hamming distance and similarity threshold selections. Hamming distance threshold defines the antibodies allowed to stay for further memory cell selection. The similarity threshold defines the antibodies allowed to be added into memory cells to become detectors. The value of the Hamming distance threshold should be adjusted empirically such that the antibodies have the ability to detect new cases correctly.

\subsection{Dynamic Training Algorithm}

One antigen (UK mandatory typeface) from each class and antibodies generated by basic clonal selection has been chosen to initialize the immune memory. After initialization, real characters were passed to a dynamic training algorithm, and the immune memory cells training and testing go hand in hand to obtain a better memory cell for classification. The clonal dynamic training algorithm is shown as Fig. 4.

While No. $<=$ size of antigens

Selected an antigen $A g$ and start to train

Classify the antigen using the current updated memory cells

If Classification strategy recognized antigen, start with another antigen

Otherwise generate antibodies $A b$ s randomly and calculate the affinity

Select $n A b$ s having the highest affinity and clone them

Apply hypermutation to the clone set $C^{i}$ to produce mutated offspring $C^{i^{*}}$

Re-calculate the HD between $A g$ and $C^{i^{*}}$. Select $A b$ s for next step

Calculate similarity between $A b$ and $A g$ Select matured $A b$ for memory cells

Stop training if the required number of matured antibodies is generated End when all antigens been trained

Fig. 4. Dynamic Training algorithm 
Classification is implemented by a fuzzy (KNN) approach, proposed by Keller in [17], which provides an improvement on existing classification techniques. For the testing, pattern was passed through the memory cells as the fuzzy KNN selected $\mathrm{k}$ closest memory cells from the immune memory. The selected memory cells were then grouped according to their class labels with the class of the largest sized group identifying the testing pattern.

\section{Experiments and Results}

Three different dataset resources 'LPR0', 'LPR1' and 'LPR2' have been collected from a car park, road, street and petrol stations within the UK. LPR0 consisted of 950 samples of licence plates that will be used for training. LPR1 and LPR2 are two datasets containing 400 samples to test the performance of the systems. Characters extracted from LPR0 data were grouped into two parts: digits and letters. The digits have 10 classes ( 0 to 9 ) and the letters have 23 classes (A to $\mathrm{Z}$ without $\mathrm{Q}, \mathrm{O}, \mathrm{I}$ ). Experiments were carried out through two different training methods. (1) Single pass training, where each antigen produces the same number of antibodies, (2) Dynamic training, described in section 3.3.

In both training, all the antibodies were first generated based on the mandatory typeface. Each antigen produced 30 antibodies before generating them from LPR0. These antigens only generated 10 antibodies each. The HD Threshold was 25 for digits and 50 for characters; the Similarity Threshold was 0.93 for digits and 0.87 for letters. The initial population for antibodies was 30 and hypermutation probability was 0.05. All parameters were determined by experimentation.

Classification results are also depended on the classification strategy. The effect of $\mathrm{k}$ in fuzzy KNN classification is examined and shows that $\mathrm{k}=5$ for the digits and $\mathrm{k}=7$ for letters gave the best performance with $\mathrm{K}=7$ giving a better combined performance. Improvement can be further achieved by dividing the letters into G1 and G2. Table 1 presents the results for both training. The results proved that dynamic training reduced the difficulties for Single pass such as, large numbers of immune memory cells, low recognition accuracy, time wasted in training and recognizing.

\section{G1: BCEJKMPSTVXZ G2: ADFGHLNRUWY}

Table 1. Two different training results for CSA

\begin{tabular}{|c|c|c|c|c|}
\hline Parameter K & \multicolumn{2}{|c|}{ Digits Accuracy \% } & \multicolumn{2}{c|}{ Letters Accuracy \% } \\
\hline & Single & Dynamic & Single & Dynamic \\
\hline K=5 & 96.5 & 98.5 & 86 & 88 \\
\hline K=7 & 95 & 96.5 & 89 & 92.4 \\
\hline K=9 & 93 & 95 & 85 & 89 \\
\hline
\end{tabular}

Table 2 presents the results for best training and testing $(\mathrm{C}=$ correct, $\mathrm{I}=$ Incorrect $)$ for our Licence Plate Character Recognition. 
Table 2. Training \& testing results for CSA

\begin{tabular}{|r|l|l|c|c|c|c|c|c|}
\hline & \multicolumn{2}{|c|}{ Training } & \multicolumn{3}{c|}{ Testing } & \multicolumn{3}{c|}{ Testing } \\
\hline Data set & \multicolumn{2}{|c|}{ LPR0 } & \multicolumn{3}{c|}{ LPR1 } & \multicolumn{3}{c|}{ LPR2 } \\
\hline & Digits & Letters & C & 1I & > 2I & C & 1I & > 2I \\
\hline Accuracy\% & 96.5 & 92.4 & 89.5 & 3.0 & 7.5 & 83.5 & 7.5 & 9.0 \\
\hline
\end{tabular}

The performance of our CS based approach has also been compared to Back Propagation Neural Networks [18]; a feed-forward neural network consisting of three layers has been employed. In this case, the multilayer perceptron (MLP) model had 256 nodes in the input layer and 20-50 in hidden layers which were determined empirically.

Letters are divided into N1, N2 so the confusion of similar characters could be corrected by Neural-Networks. The initial results using ANN for the performance of the digit network were sufficiently successful. The results are shown in table 3 .

\section{N1:ABHJLMNTUVWY N2:CDEFGKPRSXZ}

Table 3. Training \& testing results for ANN

\begin{tabular}{|c|c|c|c|c|c|c|c|c|}
\hline & \multicolumn{2}{|c|}{ Training } & \multicolumn{3}{c|}{ Testing } & \multicolumn{3}{c|}{ Testing } \\
\hline Data set & \multicolumn{2}{|c|}{ LPR0 } & \multicolumn{3}{c|}{ LPR1 } & \multicolumn{3}{c|}{ LPR2 } \\
\hline & Digits & Letters & C & 1W & >2W & C & 1W & >2W \\
\hline Accuracy \% & 94.3 & 90.5 & 84.5 & 6.0 & 9.5 & 81.0 & 9.0 & 10.0 \\
\hline
\end{tabular}

From the experiments, the clonal selection algorithm has shown that it can provide sufficient data to make generalizations from examples and can also successfully classify previously unseen examples of its training classes. Adjustments to the basic algorithm improved performance and illustrated how clonal selection could be used in LPCR.

Compared to our neural network approach the proposed AIS based method was found to be better than ANN by more than 2 percents in training and more than 3 percent in testing. The algorithm has been made to increase good candidate memory cells and reduce the training time. But the main weakness lies in the efficiency of the algorithm as the time taken to generate the memory cells could be seen to make it unattractive for time-dependent applications such as most real-world problems.

\section{Concluding Remarks}

The paper reports on the use of clonal selection algorithm for its application for Licence Plate Character Recognition. The clonal selection algorithm can be characterized as a good alternative and a more competitive approach whereby individual antibodies are competing with the whole population cooperating as an ensemble of individuals to present the final solution. The experimental results consistently show that the proposed algorithm has high classification precision. 
Moreover, when compared with BPNN the average performance of CSA is more accurate and robust. Specifically, the proposed algorithmic structure was not evaluated on a fixed location; the image is acquired manually in various views and illumination conditions in order to closely resemble real world situations.

Licence Plate Recognition is always an important research topic of artificial intelligent systems. Future work include increasing the size of the test set, improvement of the detection accuracy and classification speed, and the hybridization of CSA with Higher Order Neural Networks (HONNs) can be carried out later on.

\section{References}

1. Chang, S.L., Chen, L.S., Chung, Y.C., Chen, S.W.: Automatic licence plate recognition. IEEE Trans. Intell. Transp. Syst. 5(1), 42-53 (2004)

2. Wu, C., On, L.C., Weng, C.H., Kuan, T.S., Kengchung, N.G.: A Macao Licence Plate Recognition System. In: Proceedings of Fourth International Conference on Machine Learning and Cybernetics, Guangzhou, vol. 7, pp. 4506-4510 (2005)

3. Duan, T.D., Hong Du, T.L., Phuoc, T.V., Hoang, N.: Building an automatic vehicle licence plate recognition system. In: Proc. Int. Conf. Comput. Sci. RIVF, pp. 59-63 (2005)

4. Hu, Y., Zhu, F., Zhang, X.: A Novel Approach for License Plate Recognition Using Subspace Projection and Probabilistic Neural Network. In: Wang, J., Liao, X.-F., Yi, Z. (eds.) ISNN 2005. LNCS, vol. 3497, pp. 216-221. Springer, Heidelberg (2005)

5. Martín, F., García, M., Alba, L.: New methods for automatic reading of VLP's (Vehicle Licence Plates). In: Proc. IASTED Int. Conf. SPPRA (2002)

6. Kim, K.I., Jung, K., Kim, J.: Color Texture-Based Object Detection: An Application to License Plate Localization. In: Lee, S.-W., Verri, A. (eds.) SVM 2002. LNCS, vol. 2388, pp. 293-309. Springer, Heidelberg (2002)

7. Anagnostopoulos, C., Anagnostopoulos, I., Loumos, V., Kayafas, E.: A Licence PlateRecognition Algorithm for Intelligent Transportation System Applications. IEEE Transaction on Intelligent Transportation Systems 7(3) (September 2006)

8. Broumandnia, A., Fathy, M.: Application of pattern recognition for Farsi licence plate recognition. In: The ICGST Int. Conf. Graphics, Vision and Image Processing (GVIP), vol. 2, pp. 25-31 (2005)

9. Chang, S.-L., Chen, L.-S., Chung, Y.-C., Chen, S.-W.: Automatic Licence Plate Recognition. IEEE Transactions on Intelligent Transportation Systems 5(1) (March 2004)

10. Anagnostopoulos, C., Alexandropoulos, T., Boutas, S., Loumos, V., Kayafas, E.: A template-guided approach to vehicle surveillance and access control. In: Proc. IEEE Conf. Advanced Video and Signal Based Surveillance, pp. 534-539 (2005)

11. de Castro, L.N., Timmis, J.: Artificial Immune systems: A Novel Paradigm to Pattern Recognition. In: Corchado, A.J., Fyfe, C. (eds.) Artificial Neural Networks in Pattern Recognition, pp. 67-84. university of Paisley (2003)

12. Timmis, J., Knight, T., De Castro, L.N., Hart, E.: An Overview of Artificial Immune Systems. Natural Computation Series, 51-86 (2004)

13. Burnet, F.M.: Clonal Selection and After. Theoretical Immunology. In: Bell, G.I., Perelson, A.S., Pimbley Jr, G.H. (eds.), pp. 63-85. Marcel Dekker Inc., New York (1978)

14. de Castro, L.N., Von Zuben, F.J.: aiNet: An Artificial Immune Network for Data Analysis. In: Sacker, R.A., Newton, C.S. (eds.) Data Mining: A Heuristic Approach. Idea Publishing Group, Hershey (2001) 
15. Garain, U., Chakraborty, M., Majumder, D.: Improvement of OCR Accuracy by Similar Character Pair Discrimination: an Approach based on Artificial Immune System. In: The 18th Int. Conf. on Pattern Recognition (ICPR), vol. 2, pp. 1046-1049 (2006)

16. Garain, U., Chakraborty, M.P., Dasgupta, D.: Recognition of Handwritten Indic Script Using Clonal Selection Algorithm. In: Bersini, H., Carneiro, J. (eds.) ICARIS 2006. LNCS, vol. 4163, pp. 256-266. Springer, Heidelberg (2006)

17. Keller, J.M., Gray, M.R., Givens Jr., J.: A Fuzzy K-Nearest Neighbor Algorithm. IEEE Transactions on Systems, Man, and Cybernetics 15(4), 580-585 (1985)

18. Nijhuis, J.A.G., ter Brugge, M.H., Helmholt, K.A., Pluim, J.P.W., Spaanenburg, L., Venema, R.S., Westenberg, M.: Car licence plate recognition with neural networks and fuzzy logic. In: Proc. IEEE Int. Conf. Neural Netw., vol. 5, pp. 2232-2236 (1995) 